every $\mu \in W$, including these ergodic ones, because $S$ is minimal and every $\mu \in W$ is $t$-invariant, having therefore an invariant support set.

\title{
REFERENCES
}

[BH] J. R. Blum and L. Hanson, On invariant probability measures. I, Pacific J. Math. 10 (1960), 1125-1129.

[R] W. Rudin, Averages of continuous functions on compact spaces, Duke Math. J. 25 (1958), 195-204.

UNIVERSITY OF ROCHESTER

\section{HOMOGENEOUS NONNEGATIVE SYMMETRIC QUADRATIC TRANSFORMATIONS}

BY G. R. BLAKLEY

Communicated by J. Doob, May 19, 1964

Some recent work [2], [3] has led to almost enough knowledge about nonnegative symmetric homogeneous quadratic transformations to merit the name theory. This note presents one interesting fact, Theorem 6 , which states that in a sense almost all such transformations $\exists$ give rise to a sequence $\left\{\jmath^{0}, J^{1}, \Im^{2}, \ldots\right\}$ of iterates which converges pointwise, together with a map of the way stations leading to it. There are very few proofs of the intermediate results since, taken in their totality, they are the skeleton of the proof of Theorem 6. The articulation of this skeleton is indicated by the following scheme of dependences of theorems and lemmas.

$$
\begin{array}{ll}
\mathrm{T} 1 & \text { on } \mathrm{L} 1, \mathrm{~L} 2 . \\
\mathrm{T} 2 & \text { on } \mathrm{L} 3, \mathrm{~L} 4 . \\
\mathrm{T} 3 & \text { on } \mathrm{T} 1, \mathrm{~L} 4 . \\
\mathrm{T} 4 & \text { on } \mathrm{T} 2 . \\
\mathrm{T} 5 & \text { on } \mathrm{T} 1 . \\
\mathrm{T} 6 & \text { on } \mathrm{T} 1, \mathrm{~T} 3, \mathrm{~T} 4, \mathrm{~T} 5 .
\end{array}
$$

Let $P$ be the set of probability $n$-vectors in Euclidean $n$-space $R^{n}$ for some integer $n \geqq 2$ fixed throughout the discussion. $P^{0}$ is the set of componentwise positive probability $n$-vectors and $\partial P=P-P^{0}$. Let $\Gamma$ be a symmetric entrywise nonnegative $n$-by- $n$ matrix and de- 
fine the function $g: U \rightarrow R^{1}$ by setting $g(\eta)=(\eta, \Gamma \eta)$ for each $\eta$ belonging to the set $U=\left\{\alpha \in R^{n}\right.$ : The sum of the components of $\alpha$ is 1$\}$. Notice that $P$ is the intersection of $U$ with the nonnegative cone of $R^{n}$. The symbol $(\eta, \Gamma \eta)$ stands, of course, for the real number which is the inner (dot) product of the $n$-vector $\eta$ with the $n$-vector $\Gamma \eta$ which is the image of $\eta$ under the linear transformation naturally determined by the matrix $\Gamma$. A less usual product, the $n$-vector $\eta \Gamma \eta$, is the componentwise product of $\eta$ and $\Gamma \eta$. The homogeneous nonnegative symmetric transformation $\Im: P \rightarrow P$ maps $\eta$ to $\eta \Gamma \eta / g(\eta)$ if $g(\eta) \neq 0$ and is undefined if $g(\eta)=0$. Let $v$ be the $n$-vector all of whose components are unity and let $\theta$ be the $n$-vector all of whose components are zero. $U_{\theta}=\left\{\alpha \in R^{n}:(\alpha, v)=0\right\}$ is the translate of $U$ which contains $\theta$. If $\tau \in U_{\theta}, \eta \in U$ the Frechet derivative of $g$ with respect to $\tau$ at $\eta$ is

$$
d_{\tau} g(\eta)=\lim _{h \rightarrow 0}(g(\eta+h \tau)-g(\eta)) / h=2(\tau, \Gamma \eta) .
$$

A critical point of $g$ is a point $\eta \in U$ such that $d_{\tau} g(\eta)=0$ for each $r \in U_{\theta}$.

LEMma 1. If $\eta \in P^{0}$ and $g(\eta)>0$ then $J(\eta)=\eta$ if and only if $\eta$ is a critical point of $g$.

LEMMA 2. If $\eta \in P$ and $g(\eta)>0$ then $g(\eta) \leqq g(J(\eta))$ and $d_{\Im(\eta)-\eta} g(\eta) \geqq 0$. If, furthermore, $\eta \in P^{0}$ each equality is equivalent to the statement that $\eta$ is a critical point of $g$.

The first inequality follows from a recent result [1], the second from the Bunyakowsky-Cauchy-Schwarz inequality. The support $S(\eta)$ of $\eta$ is the set of indices of nonvanishing components of $\eta$. $g^{\eta}$ is the restriction of $g$ to the smallest coordinate hyperplane containing $\eta$ or, in other words, to the set $\left\{\alpha \in R^{n}: S(\alpha) \subset S(\eta)\right\}$. It is easy to see how these lemmas lead to the fundamental

THEOREM 1. If $\eta \in P$ and $g(\eta)>0$ then $g(\eta) \leqq g(J(\eta)), d_{\Im(\eta)-\eta} g(\eta) \geqq 0$, and the following four conditions are equivalent:

(i) $\eta=\mathfrak{J}(\eta)$,

(ii) $\eta$ is a critical point of $g^{\eta}$,

(iii) $g(\eta)=g(J(\eta))$,

(iv) $d_{\zeta(\eta)-\eta} g(\eta)=0$.

Note that if $\eta \in P$ and $g(\eta) \neq 0$ it follows that the sequence $\left\{\eta, J(\eta), J^{2}(\eta), \ldots\right\}$ of $n$-vectors is well defined and that the associated sequence $\left\{g(\eta), g(J(\eta)), g\left(J^{2}(\eta)\right), \cdots\right\}$ of positive real numbers is in fact monotone nondecreasing. 
LEMMA 3. $\Gamma \eta=g(\eta) \cup$ if $\eta$ is a critical point of $g$.

A flat is a translate of a subspace of $R^{n} \cdot g$ is a cylinder along a flat $F$ if it is constant on each translate of $F$.

Lemma 4. If the set FCU of critical points of $g$ is nonvoid it is a flat and $g$ is a cylinder along $F$.

THEOREM 2. If $\Gamma$ is nonsingular there is at most one critical point of $g$.

PROOF. If $\alpha, \beta$ are critical points of $g$ then $g(\alpha)=g(\beta)=g^{*}$ and $\Gamma \alpha=\Gamma \beta=g^{*} v$. If $\Gamma$ is nonsingular then $\alpha=\beta$.

THEOREM 3. $g$ is constant on each component of the fixed point set of $\mathfrak{J}$.

$\alpha$ dominates $\beta$ if $\alpha \neq \beta$ and $\alpha-\beta$ is a componentwise nonnegative $n$-vector. If $\Gamma$ is singular and there are no $\alpha, \beta \in P$ for which $\Gamma \alpha$ dominates $\Gamma \beta$, call $\Gamma$ perfectly balanced. A principal submatrix $\Gamma^{*}$ of $\Gamma$ is one obtained by striking out rows and columns with the same indices. Each principal submatrix $\Gamma^{*}$ of $\Gamma$ must be symmetric since $\Gamma$ is. If $\alpha$ is a vector in $R^{n}$ whose support contains $n^{*}$ integers, let $\Gamma^{*}=\Gamma(S(\alpha))$ be the $n^{*}$-by- $n^{*}$ principal submatrix of $\Gamma$ whose entries are those entries of $\Gamma$ determined by row and column indices both of which belong to $S(\alpha)$.

THEOREM 4. If $\lambda, \mu$ are distinct critical points of $g^{\lambda}$ which have the same support $S(\lambda)=S(\mu)$ then $\Gamma(S(\lambda))$ is perfectly balanced.

Proof. It suffices to show that $\Gamma$ is perfectly balanced if there are distinct points $\lambda, \mu$ of $P^{0}$ which are critical points of $g . \Gamma$ is singular in such a case. If there were $\alpha, \beta \in P$ such that $\Gamma \alpha$ dominates $\Gamma \beta$ then $0<(\lambda, \Gamma \alpha-\Gamma \beta)=(\lambda, \Gamma(\alpha-\beta))$, which would imply that $\lambda$ is not a critical point of $g$ after all.

An argument from the continuity of $g$ where it is nonzero and the monotonicity of a sequence $\left\{g(\eta), g(J(\eta)), g\left(J^{2}(\eta)\right), \ldots\right\}$ gives

THEOREM 5. $\lambda$ is a critical point of $g^{\lambda}$ if $\lambda$ is a limit point of a sequence $\left\{\eta, J(\eta), J^{2}(\eta), \cdots\right\}$.

THEOREM 6. If there is no nonzero perfectly balanced principal submatrix $\Gamma^{*}$ of $\Gamma$ each component of the fixed point set of $J$ is a singleton. Thus $\left\{\eta, J(\eta), J^{2}(\eta), \cdots\right\}$ converges at each $\eta \in P$ for which $g(\eta)>0$.

Proof. Let $\lambda$ be any fixed element of the component $C$ of $I=\{\eta: J(\eta)=\eta\}$. If $\mu \in C$ then $g(\mu)=g(\lambda)>0$ in consequence of Theorem 3. If $C$ were not a singleton it would be infinite and would therefore contain two distinct points $\alpha, \beta$ such that $S(\alpha)=S(\beta)$. The prin- 
cipal submatrix $\Gamma^{*}=\Gamma(S(\alpha))$ would have a nonzero entry since $g(\alpha)=g(\lambda)>0$. It would then follow from Theorem 1 that $\alpha$ and $\beta$ would be critical points of $g^{\alpha}$ and thus from Theorem 4 that $\Gamma^{*}$ would be perfectly balanced, contrary to assumption. Hence each component $C$ of $I$ is a singleton. Let $\eta \in P$. Theorem 5 , taken in conjunction with Theorem 1 , states that all limit points of $\left\{\eta, J(\eta), J^{2}(\eta), \cdots\right\}$ belong to $I$. It is not hard to show that $\left\{\eta, J(\eta), J^{2}(\eta), \cdots\right\}$ cannot have limit points in two different components of $I$. Thus all limit points of $\left\{\eta, J(\eta), J^{2}(\eta), \cdots\right\}$ lie in precisely one component $C$ of $I$ and $C$ is a singleton.

These are highlights of the symmetric homogeneous quadratic theory. It has extensions to the theory of differential equations as well as more general types of quadratic transformations, to higher degree transformations and to the nonnegative nonsymmetric theory whose absence Ulam refers to in [4]. In fact the symmetric theory plays a crucial role in the more general theory even though there is, on the surface, scant similarity [3] between symmetric and nonsymmetric quadratic transformations. [2], [3] and their sequels take these topics up in more detail.

\section{REFERENCES}

1. G. R. Blakley and Prabir Roy, A Hölder type inequality for symmetric matrices with nonnegative entries (in preparation).

2. G. R. Blakley, The sequence of iterates of a nonnegative nonlinear transformation. $I$ (in preparation).

3. G. R. Blakley and R. D. Dixon, The sequence of iterates of a nonnegative nonlinear transformation. II (in preparation).

4. M. T. Menzel, P. R. Stein and S. M. Ulam, Quadratic transformations, Part I, Los Alamos Scientific Laboratory Report LA-2305, Physics and Mathematics (TID4500, 14th edition), distributed May 29, 1959.

UNIVERSITY OF ILLINOIS 